\title{
Sensitive torsional magnetometer for use with small samples: The 2DEG magnetisation revisited
}

\author{
S.A.J. Wiegers ${ }^{\mathrm{a}, *}$, J.C. Maan ${ }^{\mathrm{a}}$, C.T. Foxon ${ }^{\mathrm{b}, 1}$ \\ a High Field Magnet Laboratory and Research Institute for Materials, University of Nijmegen, Toernooiveld I, \\ NL-6525 ED Nijmegen, The Netherlands \\ ${ }^{\mathrm{b}}$ Philips Research Laboratories, Redhill, Surrey, UK
}

\begin{abstract}
We have constructed a torsional magnetometer for use with small samples. To increase the sensitivity six sets of electrodes are present instead of one. First results have been obtained on the magnetisation of a single layer, high mobility AlGaAs-GaAs heterojunction 2DEG between 2 and $4 \mathrm{~K}$.
\end{abstract}

\section{Introduction}

Torsional magnetometers offer some advantages over other methods of measuring magnetisation: the more important considerations being sensitivity and fit for use in high, inhomogeneous magnetic fields. Even for liquid samples torsional magnetometers have been developed in recent years (see the paper by Van Steenbergen et al. in these proceedings). A drawback is the sometimes large and unpredictable background signal of such a device which masks a small superimposed magnetisation signal.

A torsional magnetometer measures the torque or the force that is exerted on a magnetic moment in a nonparallel magnetic field or a field gradient. Usually the torque or force is transduced into a movement that is capacitively detected. The magnetometer discussed in this paper consists of a rotatable, thin $(0.2 \mathrm{~mm})$ hysol disk, suspended through the axis by a $50 \mu \mathrm{m}, 1 \mathrm{~cm}$ long manganin wire, similar to the type used by Eisenstein et al. [1]. However, in this case six electrodes have been silver painted onto the disk, which are facing and semi-

\footnotetext{
* Corresponding author.

${ }^{1}$ Present address: Department of Physics, University of Nottingham, Nottingham MG7 2RD, UK.
}

overlapping two other sets of six electrodes on a similar, but fixed disk. The gap between the two disks is about $0.2 \mathrm{~mm}$. The sample is mounted on a small hysol table glued in the centre of the moving disk. A single-turn, $30 \mu \mathrm{m} \mathrm{Cu}$ wire coil is wound around the table for calibration purposes.

Small samples are particularly well suited for magnetisation measurements in torsional magnetometers because they negligibly perturb the mechanical constants of the device. This is important for the mechanical eigenfrequency that should be as high as possible to spectrally separate the almost DC measurement signal and the AC rotational eigenmode. We have obtained a high eigenfrequency $(25 \mathrm{~Hz})$ by choosing light-weight moving electrodes and a large spring constant. The sensitivity loss due to the high spring constant has been balanced by having multiple electrodes, resulting in a sensitivity of about $20 \mathrm{pN} \mathrm{m}$. By using the least amount of material for the moving disk the background signal has been minimised as well as any gravitational unbalance which will couple rotations of the disk to vertical and sideway movements of the whole magnetometer.

A good example of a small magnetisation signal is given by the 2DEG, the smallness being due to its $2 \mathrm{D}$ geometry. The magnetisation of the 2DEG should provide 
important information concerning the DOS of these systems, which is held responsible for the existence of the QHE. The magnetisation of a 2DEG has been calculated by Zawadski [2] and MacDonald et al. [3]. Experimental results have been reported by Eisenstein et al. [1].

\section{Experimental results}

Our first results have been obtained with a single layer (area $\left.0.25 \mathrm{~cm}^{2}\right)$, high mobility $\left(\mu=8.7 \times 10^{5} \mathrm{~cm}^{2} / \mathrm{V} \mathrm{s}\right.$ ) 2DEG formed in an AlGaAs-GaAs heterojunction having a carrier density of $3.8 \times 10^{11} \mathrm{~cm}^{-2}$. The sample, mounted at an angle $\theta$ with respect to the magnetic field, produces a magnetisation that is nearly proportional to the field and does not depend much on temperature between 2 and $4 \mathrm{~K}$. After subtracting this linear magnetisation from the signal we obtain the result shown in Fig. 1.

We observe at certain magnetic fields small and reproducible peaks in the magnetometer signal. The ratios of the magnetic fields at which they occur, are 1,2 and 3 to better than $10^{-3}$, and correspond to filling factors $v=2$, 4 and 6 . At $4 \mathrm{~K}$ we observe only $v=2$ and 4 , but at $2 \mathrm{~K}$ $v=6$ is visible as well. The $v=2$ signal between $T=2$ and $4 \mathrm{~K}$ is shown in Fig. 2. The amplitude as well as the width of the structures increase with decreasing temperature between 2 and $4 \mathrm{~K}$.

The rate at which the magnetic field is swept does not influence the structures observed at $v=4$ and 6 . The $v=2$ structure at $2 \mathrm{~K}$ does show a strong dependence on the sweeprate and direction: it is a peak or dip, depending

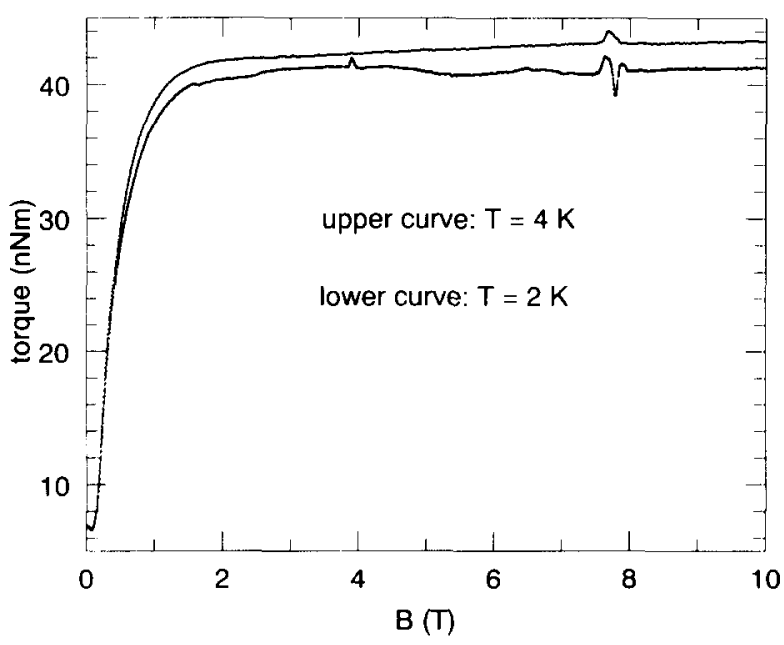

Fig. 1. Magnetometer signal at 4 and $2 \mathrm{~K}$ with $2 \mathrm{DEG}$ sample mounted. A linear magnetisation background has been subtracted.

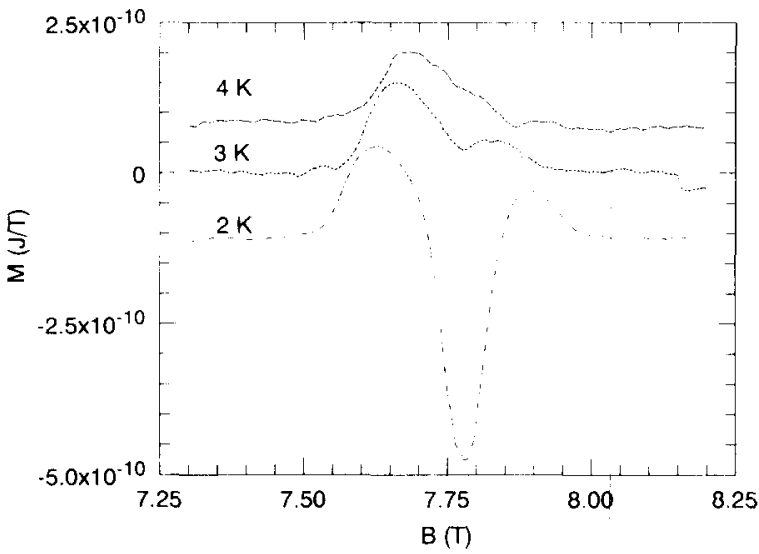

Fig. 2. The temperature dependence of the $v=2$ structure: $T=4 \mathrm{~K}, T=3 \mathrm{~K}$ and $T=2 \mathrm{~K}$. The sweeprate is $7.5 \mathrm{mT} / \mathrm{s}$.

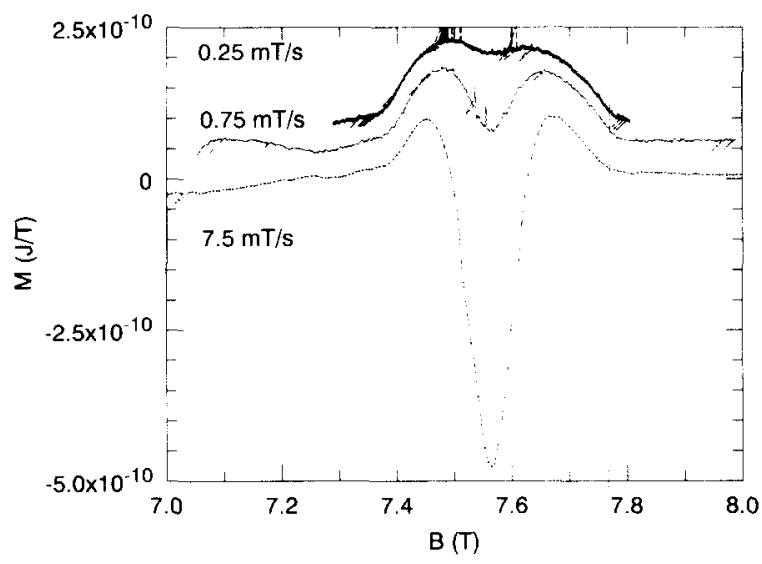

Fig. 3. The sweeprate dependence of the $v=2$ structure: 7.5 , 0.75 and $0.25 \mathrm{mT} / \mathrm{s}$.

on the direction of the sweep, and its magnitude is proportional to the sweeprate. However, for slow enough sweeprates the $v=2$ structure converges to a peak that has approximately the same shape as at $v=4$ and 6 . This behaviour is shown in Fig. 3.

After illumination with a LED for more than $10 \mathrm{~ms}$ the structures have disappeared altogether, and the magnetometer signal is drastically changed. This point is still under investigation.

\section{Discussion}

The observed linear magnetisation contribution stems from the AlGaAs-GaAs substrate, while the structures at 
even filling factors are clearly due to the 2DEG. We consider first the low sweeprate limit. The increase of the magnitude and width of the structures as the temperature is decreased, resembles the behaviour of the depth of the resistance minima and the width of the Hall plateaux in the QHE. Furthermore, the magnetisation equivalent of the structure at $v=2$ is about $100 \mathrm{pJ} / \mathrm{T}$. This is much larger than the maximum Landau diamagnetic contribution, which (using an effective mass of $0.066 m_{e}$ ) for our sample is only $10 \mathrm{pJ} / \mathrm{T}$. Note that our sensitivity of $20 \mathrm{pNm}$ is enough to detect this $10 \mathrm{pJ} / \mathrm{T}$ at $B=8 \mathrm{~T}$.

The observed sweeprate-dependent contribution is due to eddy currents in the 2DEG as $\rho_{x x}$ comes sufficiently close to zero. We estimate from our data at $2 \mathrm{~K}$ and $v=2$ for $\rho^{\prime}$ (resistivity) about $2 \mu \Omega$. This is another indication that the observed structures occur exactly at some integer filling factor, as well as that the sample is of good quality.
In conclusion, we do not observe the behaviour of the magnetisation measured by Eisenstein et al. [1] and calculated by Zawadski et al. [2] and MacDonald et al. [3]: a sawtooth like curve with steps at even filling factors. A possible cause may be that our sample mobility is better by a factor of three for their single-layer sample and by a factor of $\mathbf{1 0}$ for their 50 layer sample. By performing measurements at lower temperatures we expect to obtain more information.

\section{References}

[1] J.P. Eisenstein, H.L. Stoermer, V. Narayanamurti, A.Y. Cho, A.C. Gossard and C.W. Tu, Phys. Rev. Lett. 55 (1985) 875.

[2] W. Zawadski and R. Lassnig, Surf. Sci. 142 (1984) 225.

[3] A.H. MacDonald, H.C.A. Oji and K.L. Liu, Phys. Rev. B 34 (1986) 2681. 